\title{
Teleconsultoria na atenção primária no norte de Minas Gerais: cenário e fatores associados à sua não utilização por médicos
}

\author{
Teleconsultation at primary health care in north of Minas Gerais: scenario \\ and factors associated with its non-utilization by physicians
}

\section{Teleconsultoría en la atención primaria en el norte de Minas Gerais: escenario y factores asociados a la no utilización suya por médicos}

\section{Renata Fiúza Damasceno ${ }^{1, a}$}

damascenoenf@gmail.com | https://orcid.org/o0oo-0002-9525-8527

\author{
Antônio Prates Caldeira ${ }^{1, b}$ \\ antonio.caldeira@unimontes.br | https://orcid.org/o0oo-0002-9990-9083
}

\author{
${ }^{1}$ Universidade Estadual de Montes Claros. Montes Claros, MG, Brasil. \\ a Mestrado em Ciências da Saúde pela Universidade Estadual de Montes Claros. \\ b Doutor em Ciências da Saúde pela Universidade Federal de Minas Gerais.
}

\section{Resumo}

Este artigo objetiva descrever o cenário e identificar fatores associados à não utilização da teleconsultoria pelos médicos da atenção primária na macrorregião Norte de Minas Gerais. Trata-se de um estudo transversal. Para a coleta de dados, foi direcionado um questionário autoaplicado, previamente testado, aos gestores municipais de todos os municípios dessa área. Após análises bivariadas para identificação dos fatores associados à não utilização da teleconsultoria, foi empregada regressão de Poisson com variância robusta. Participaram do estudo 86 gestores municipais de saúde. Importantes barreiras estruturais à implementação da teleconsultoria foram identificadas: falta de computadores com acesso à internet nas unidades básicas de saúde $(76,7 \%)$ e a qualidade insatisfatória de conexão da internet (16,3\%). Na análise múltipla dos fatores associados, apenas a falta de estratégias de motivação por parte dos gestores mostrouse associada ( $\mathrm{p}<0,007 ; \mathrm{RP}=2,19$; IC-95\%:1,24-3,86) à não utilização da teleconsultoria. Os resultados reforçam o papel do gestor de saúde na promoção do uso do serviço de teleconsultoria na atenção primária.

Palavras-chave: Telemedicina; Atenção primária à saúde, Gestor de saúde; Estratégias de motivação; Barreiras estruturais. 


\begin{abstract}
This paper aims to describe the scenario and to identify factors associated with non-use of teleconsultation by primary care physicians in the macrorregião Norte de Minas Gerais (Northern macroregion of Minas Gerais), Brazil. This study is a cross-sectional one. For data collection, a self-administered questionnaire was fill in by the health managers working in all the municipalities of that macroregion; it was previously tested through some of those professionals. The Poisson regression with robust variance was used after bivariate analyzes to identify factors associated with non-use of teleconsultation.To participate in the study 86 municipal health managers were invited. Important structural barriers to teleconsultation implementation were identified: lack of computers with access to internet in basic health units (76.7\%) and poor internet connection quality (16.3\%). After multiple analysis, only the lack of motivation strategies by the health managers was associated ( $\mathrm{p}<0.007 ; \mathrm{PR}=2.19$; IC95\%:1.24-3.86) with non-use of teleconsultation by physicians. The results reinforce the role played by the health manager in promoting the use of teleconsultation service in primary care.
\end{abstract}

Keywords: Telemedicine; Primary health care; Health manager; Motivation strategies; Structural barriers.

\begin{abstract}
El objetivo de este artículo es describir el escenario y identificar factores asociados a la no utilización de la teleconsultoría por los médicos de la atención primaria en la macrorregião Norte de Minas Gerais (macrorregión Norte de Minas Gerais), Brasil. Se trata de un estudio transversal. Para la recolección de datos, se utilizó un cuestionario autoadministrado, contestado por gestores de salud que trabajan en todos los municipios de la región; el cuestionario ha sido previamente puesto a prueba con la colaboración de algunos de esos gestores. La regresión de Poisson con varianza robusta fue empleada después de análisis bivariados para identificar los factores asociados a la no utilización de la teleconsultoria. Participaron del estudio 86 gestores municipales de salud. Se identificaron importantes barreras estructurales a la implementación de la teleconsultoria: falta de computadoras con acceso a internet en las unidades básicas de salud (76,7\%) y mala calidad de conexión a internet (16,3\%). Después del análisis múltiple, sólo la falta de estrategias de motivación por parte de los gestores de salud se asoció ( $\mathrm{p}<0,007 ; \mathrm{RP}=2,19 ;$ IC95\%:1,24-3,86) con el no uso de la teleconsultoría. Los resultados refuerzan el papel del gestor de salud en la promoción del uso del servicio de teleconsultoría en la atención primaria.
\end{abstract}

Palabras clave: Telemedicina; Atención primaria a la salud; Gestor de salud; Estrategias de motivación; Barreras estructurales.

INFORMAÇÕES DO ARTIGO

Contribuição dos autores:

Concepção e desenho do estudo: Renata Fiúza Damasceno e Antônio Prates Caldeira.

Aquisição, análise ou interpretação dos dados: Renata Fiúza Damasceno e Antônio Prates Caldeira.

Redação do manuscrito: Renata Fiúza Damasceno e Antônio Prates Caldeira.

Revisão crítica do conteúdo intelectual: Renata Fiúza Damasceno e Antônio Prates Caldeira.

Declaração de conflito de interesses: Nenhum conflito de interesses a declarar.

Fontes de financiamento: Não houve.

Considerações éticas: O projeto foi aprovado pelo Comitê de Ética em Pesquisa da Universidade Estadual de Montes Claros sob parecer número 1.416.827/2016.

Agradecimentos/Contribuições adicionais: Não há.

Histórico do artigo: Submetido: 04.jun.2017 | Aceito: 03.dez.2018 | Publicado: 21.dez.2018.

Apresentação anterior: Não houve.

Licença CC BY-NC atribuição não comercial. Com essa licença é permitido acessar, baixar (download), copiar, imprimir, compartilhar, reutilizar e distribuir os artigos, desde que para uso não comercial e com a citação da fonte, conferindo os devidos créditos de autoria e menção à Reciis. Nesses casos, nenhuma permissão é necessária por parte dos autores ou dos editores. 


\section{Introdução}

No Brasil, a organização da atenção à saúde e a ampliação do acesso dos cidadãos aos serviços ofertados pelo Sistema Único de Saúde (SUS) constituem os principais desafios para os gestores de saúde nas três esferas de governo: municipal, estadual e federal. Para os gestores municipais, que destacam a insuficiente oferta de atenção especializada como um dos obstáculos à constituição de rede, superar os efeitos da fragmentação que persistem na atenção à saúde do SUS e potencializar a Atenção Primária à Saúde (APS) como porta de entrada preferencial e centro ordenador e integrador das redes de serviços é um dos principais desafios ${ }^{1}$.

Nesse contexto, a telessaúde, importante componente no fortalecimento da APS e organização das redes de atenção, pode ser uma das estratégias para superação desse desafio. Trata-se de uma grande inovação no nível tecnológico, social e cultural, que tem o potencial de aumentar o acesso e a qualidade dos serviços de saúde e reduzir os gastos do sistema de saúde ${ }^{2,3}$. O termo telessaúde (ou telemedicina ou e-Saúde) vem sendo usado para designar as atividades que utilizam as tecnologias de informação e comunicação na atenção à saúde ${ }^{4}$. Vários termos e conceitos podem ser encontrados para definir telessaúde. Porém, o princípio central é a utilização de Tecnologias de Informação e Comunicação (TIC) na saúde, quando os atores envolvidos estão distantes um do outro em seus diferentes níveis (primário, secundário e terciário) 5 .

Entre as diversas modalidades da telessaúde, a teleconsultoria e os serviços de telediagnóstico são as modalidades com maior aplicação nos países em desenvolvimento, por serem de baixo custo, exigirem menor infraestrutura de comunicação e propiciarem real economia para o sistema de saúde ${ }^{6}$. No Brasil, o modelo de teleconsultoria assíncrono (off-line) é o mais utilizado e tem grande aplicação em regiões remotas, pois permite utilizar equipamentos e sistemas simples que necessitam que necessitam de baixa velocidade de transmissão da internet ${ }^{7}$.

O Norte de Minas Gerais compreende uma macrorregião de saúde com 86 municípios e nove regiões de saúde, com população total de pouco mais de 1,5 milhão de habitantes. É uma região caracterizada por possuir baixa densidade demográfica, forte desigualdade social, grandes distâncias entre municípios e extensas áreas rurais $^{8}$, onde, portanto, o uso do serviço de teleconsultoria pelos profissionais médicos que atuam na APS pode ampliar o acesso da população a serviços especializados e melhorar a qualidade do atendimento, com impactos positivos na atenção à saúde. Trata-se, portanto, de uma região emblemática para avaliação da utilização e impacto dos serviços de teleconsultoria e do papel desempenhado pelos diversos atores envolvidos.

Na estrutura de gestão efuncionamento do Programa de Telessaúdeno Brasil(Programa Nacional Telessaúde Brasil Redes), o papel do gestor municipal de saúde é fundamental para o fortalecimento do programa, visto que cabe a ele comprometer-se com a implementação, monitoramento e avaliação do mesmo no seu respectivo município, além de promover a integração dos profissionais de saúde às ações do Telessaúde ${ }^{9}$. Considerando que os estudos sobre telessaúde comumente são realizados junto aos usuários dos serviços - profissionais de saúde - o presente estudo tem por objetivo descrever o cenário e identificar os fatores relacionados ao município e ao gestor municipal de saúde associados à não utilização do serviço de teleconsultoria pelos médicos que atuam na atenção primária na macrorregião de saúde Norte de Minas Gerais.

\section{Métodos}

Trata-se de um estudo transversal, conduzido de março a agosto de 2016 na macrorregião de saúde Norte de Minas Gerais, Brasil. Para coleta de dados, foi utilizado questionário autoaplicado com 28 questões agrupadas em quatro blocos: caracterização do gestor municipal de saúde e da estrutura informacional das Unidades Básicas de Saúde (UBS); acesso a serviços de saúde especializados; fatores associados ao uso do serviço de teleconsultoria; satisfação com o serviço de teleconsultoria. As características do município informadas pelos gestores foram confirmadas no sítio eletrônico do Instituto Brasileiro de Geografia e Estatística (IBGE). 
Inicialmente, foi realizado um estudo piloto para testar o instrumento de coleta de dados com a participação de cinco gestores municipais de saúde. Como não houve necessidade de reformulação e adequações do questionário, os gestores que participaram nessa etapa foram incluídos no estudo principal.

A análise estatística foi realizada por meio do programa Statistical Package for the Social Sciences (SPSS), versão 20.o (SPSS for Windows, Chicago, EUA). A associação entre variáveis relacionadas ao município e ao gestor de saúde e a não utilização do serviço de teleconsultoria pelos médicos da APS foi investigada por meio do teste qui-quadrado ou teste exato de Fisher. As variáveis que se mostraram associadas até o nível de $20 \%$ foram avaliadas conjuntamente por meio da análise de regressão de Poisson, com variância robusta ${ }^{10}$. Nessa etapa, foram obtidas as razões de prevalências (RP) e seus respectivos intervalos de confiança de $95 \%$ (IC 95\%), sendo adotado para o modelo final o nível de significância de $5 \%(\mathrm{p}<0,05)$.

Todos os participantes foram orientados sobre o estudo e apresentaram sua anuência por meio da assinatura do Termo de Consentimento Livre e Esclarecido. O projeto de pesquisa teve consentimento institucional da Superintendência Regional de Saúde, vinculada à Secretaria de Estado de Saúde de Minas Gerais.

\section{Resultados}

Dos 86 (100\%) gestores municipais de saúde da macrorregião Norte de Minas Gerais que participaram deste estudo, 90,7\% atuavam como secretários municipais de saúde e 9,3\% como coordenadores municipais da APS, 51,2\% deles eram do sexo masculino e a idade do grupo variou de 25 a 67 anos, com média de 40,2 (DP $\pm 10,22)$ anos. A maioria relatou ter ensino superior completo $(74,4 \%)$ e metade $(50 \%)$ informou trabalhar como gestor municipal de saúde há menos de três anos.

Quanto à caracterização do cenário norte-mineiro de utilização do serviço de teleconsultoria pelos médicos de equipes da APS, mais da metade dos municípios da região apresentaram população com até 10 mil habitantes (59,3\%); em apenas três municípios (3,5\%), o Índice de Desenvolvimento Humano Municipal (IDHM), que retrata a qualidade de vida de uma população, era alto (entre 0,700 e 0,799$)^{11}$; e a maioria dos municípios (59,3\%) localiza-se a mais de 100 quilômetros do município de referência para atenção especializada (Tabela 1).

Tabela 1 - Caracterização do cenário norte-mineiro relativo à utilização do serviço de teleconsultoria pelos médicos na atenção primária à saúde (APS) - 2016

\begin{tabular}{lcc}
\hline Variável & n & (continua) \\
\hline Porte populacional (Número de habitantes) & & \\
$\leq 5.000$ & 15 & 17,4 \\
5.001 até 10.000 & 36 & 41,9 \\
10.001 até 20.000 & 15 & 17,4 \\
20.001 até 50.000 & 15 & 17,4 \\
50.001 até 100.000 & 4 & 4,7 \\
100.001 até 500.000 & 1 & 1,2 \\
IDHM & & \\
Alto & 3 & 3,5 \\
Médio & 63 & 73,3 \\
Baixo & 20 & 23,3
\end{tabular}




\begin{tabular}{|c|c|c|}
\hline Variável & $\mathbf{n}$ & $(\%)$ \\
\hline \multicolumn{3}{|l|}{ Tipo de conexão à internet } \\
\hline Cabo & 23 & 26,8 \\
\hline Rádio & 37 & 43,0 \\
\hline Via satélite & 15 & 17,4 \\
\hline Sem informação/Não sabe responder & 11 & 12,8 \\
\hline \multicolumn{3}{|l|}{ Qualidade da conexão à internet } \\
\hline Ótima/Boa & 40 & 46,5 \\
\hline Variável & $\mathrm{n}$ & $(\%)$ \\
\hline Satisfatória & 32 & 37,2 \\
\hline Ruim & 11 & 12,8 \\
\hline Péssima & 3 & 3,5 \\
\hline \multicolumn{3}{|l|}{ Disponibilidade de computador com acesso à internet nas UBS } \\
\hline Em todas as UBS & 20 & 23,3 \\
\hline Na maioria das UBS & 31 & 36,0 \\
\hline Em menos da metade das UBS & 13 & 15,1 \\
\hline Em pelo menos uma UBS & 20 & 23,3 \\
\hline Em nenhuma UBS & 2 & 2,3 \\
\hline \multicolumn{3}{|l|}{ Distância até município que recebe maior número de encaminhamentos } \\
\hline Até 50 km & 7 & 8,1 \\
\hline 51 a $100 \mathrm{~km}$ & 27 & 31,4 \\
\hline 101 a $250 \mathrm{~km}$ & 39 & 45,3 \\
\hline 251 a $500 \mathrm{~km}$ & 12 & 14,0 \\
\hline Não se aplica & 1 & 1,2 \\
\hline \multicolumn{3}{|l|}{ Uso do serviço de teleconsultoria pelos profissionais médicos da APS } \\
\hline Sim & 59 & 68,6 \\
\hline Não & 27 & 31,4 \\
\hline \multicolumn{3}{|l|}{ Fatores limitantes do uso de teleconsultoria pelos médicos na APS } \\
\hline Interesse do professional médico & 30 & 34,9 \\
\hline Acesso a computador com internet na UBS & 21 & 24,4 \\
\hline Tempo do profissional médico durante o seu horário de trabalho & 15 & 17,4 \\
\hline Treinamento para uso do serviço & 10 & 11,6 \\
\hline Informação sobre o serviço & 7 & 8,1 \\
\hline Falta de recomendação por parte da Secretaria Municipal de Saúde & 3 & 3,5 \\
\hline
\end{tabular}

Fonte: Os autores (2016).

Em relação ao acesso a serviços de saúde especializados, a quantidade de encaminhamento de pacientes da APS para consultas especializadas foi considerada alta pela maioria dos gestores (83,7\%). Além disso, mais de $88 \%$ deles apontaram ter dificuldade para garantir o acesso dos pacientes a essas consultas, sendo que o tempo de espera para acesso às mesmas variou de um a três meses em 41,9\% dos municípios e de quatro a mais de doze meses em $40,7 \%$.

No que se refere à estrutura informacional das UBS, registra-se que, apesar de 98,8\% dos gestores municipais de saúde considerarem que a presença de computador com acesso à internet ajuda o trabalho dos profissionais da APS, apenas 23,3\% dos municípios contavam com computadores com essa característica em todas as unidades e somente $4,7 \%$ possuíam qualidade da internet local referida como ótima. Para a 
maioria dos gestores (67,4\%), o que mais dificulta a disponibilização de computador com acesso à internet para as UBS é recurso financeiro insuficiente para aquisição e manutenção.

Cabe destacar que não há utilização do serviço de teleconsultoria pelos profissionais médicos atuantes na APS em mais de 30\% dos municípios (31,4\%). De acordo com os gestores, os principais fatores limitantes do uso desse serviço pelos médicos na APS são a falta de interesse dos profissionais médicos e a pouca disponibilidade de acesso a computador com internet na UBS (Tabela 1).

Nesse contexto, ressalta-se que oito (9,3\%) gestores informaram desconhecer o serviço de teleconsultoria e 43 (50,0\%) tinham alguma dúvida sobre o funcionamento do mesmo. A maioria dos gestores $(74,4 \%)$ informou utilizar estratégias para motivar o uso do serviço de teleconsultoria pelos profissionais médicos que atuam na APS, entre as quais foram citadas a melhoria das condições de trabalho e o estabelecimento e acompanhamento de metas.

A Tabela 2 apresenta os resultados da análise bivariada para associações entre variáveis relacionadas ao município e ao gestor municipal de saúde com a não utilização do serviço de teleconsultoria pelos profissionais médicos que atuam na APS. Após análise múltipla, permaneceu no modelo final apenas a variável 'falta de estratégias de motivação para uso do serviço' (Tabela 3).

Tabela 2 - Variáveis associadas à não utilização do serviço de teleconsultoria pelos profissionais médicos na atenção primária à saúde (APS). Macrorregião Norte de Minas Gerais, Brasil 2016 (Análise bivariada)

(continua)

\begin{tabular}{|c|c|c|c|c|c|}
\hline \multirow[t]{3}{*}{ Variáveis } & \multicolumn{4}{|c|}{ Utiliza teleconsultoria } & \multirow[b]{3}{*}{ p-valor } \\
\hline & \multicolumn{2}{|c|}{ Não } & \multicolumn{2}{|c|}{ Sim } & \\
\hline & (n) & $(\%)$ & (n) & $(\%)$ & \\
\hline
\end{tabular}

\section{Relacionadas ao município}

\begin{tabular}{|c|c|c|c|c|c|}
\hline Porte populacional & & & & & 0,640 \\
\hline$\leq 10.000$ hab. & 17 & 63,0 & 34 & 57,6 & \\
\hline >10.000 hab. & 10 & 37,0 & 25 & 42,4 & \\
\hline IDHM & & & & & 0,878 \\
\hline Baixo & 6 & 22,2 & 14 & 23,7 & \\
\hline Alto/Médio & 21 & 77,8 & 45 & 76,3 & \\
\hline Distância de centros de referência & & & & & 0,996 \\
\hline$>100 \mathrm{~km}$ & 16 & 59,3 & 35 & 59,3 & \\
\hline$\leq 100 \mathrm{~km}$ & 11 & 40,7 & 24 & 40,7 & \\
\hline Qualidade da internet & & & & & $0,757^{*}$ \\
\hline Insatisfatória & 5 & 18,5 & 9 & 15,3 & \\
\hline Satisfatória & 22 & 81,5 & 50 & 85,7 & \\
\hline Computadores com internet nas UBS & & & & & 0,158 \\
\hline Menos da metade & 8 & 29,6 & 27 & 45,8 & \\
\hline Quase todas ou todas & 19 & 70,4 & 32 & 54,2 & \\
\hline \multicolumn{6}{|l|}{ Relacionadas ao gestor de saúde } \\
\hline Sexo & & & & & 0,399 \\
\hline Masculino & 12 & 44,4 & 32 & 54,2 & \\
\hline Feminino & 15 & 55,6 & 27 & 45,8 & \\
\hline Escolaridade & & & & & 0,561 \\
\hline Médio/superior incompleto & 8 & 29,6 & 14 & 23,7 & \\
\hline Superior completo & 19 & 70,4 & 45 & 76,3 & \\
\hline
\end{tabular}


(conclusão)

\begin{tabular}{|c|c|c|c|c|c|}
\hline \multirow[t]{3}{*}{ Variáveis } & \multicolumn{4}{|c|}{ Utiliza teleconsultoria } & \multirow[b]{3}{*}{ p-valor } \\
\hline & \multicolumn{2}{|c|}{ Não } & \multicolumn{2}{|c|}{ Sim } & \\
\hline & (n) & $(\%)$ & (n) & $(\%)$ & \\
\hline Experiência na gestão em saúde & & & & & 0,435 \\
\hline$\leq 5$ anos & 23 & 85,2 & 46 & 78,0 & \\
\hline$>5$ anos & 4 & 14,8 & 13 & 22,0 & \\
\hline Sabe como funciona o serviço de teleconsultoria & & & & & 0,009 \\
\hline Não & 9 & 33,3 & 6 & 10,2 & \\
\hline Sim & 18 & 66,7 & 53 & 89,8 & \\
\hline Recomenda o uso aos médicos da APS & & & & & $0,003 *$ \\
\hline Não & 8 & 29,6 & 3 & 5,1 & \\
\hline Sim & 19 & 70,4 & 56 & 94,9 & \\
\hline Estratégias de motivação para uso do serviço & & & & & $<0,001$ \\
\hline Não & 14 & 51,9 & 8 & 13,6 & \\
\hline $\mathrm{Sim}$ & 13 & 48,1 & 51 & 86,4 & \\
\hline
\end{tabular}

Fonte: Os autores (2016).

${ }^{(*)}$ Teste exato de Fisher

Tabela 3 - Variáveis associadas à não utilização do serviço de teleconsultoria pelos profissionais médicos na atenção primária à saúde (APS). Macrorregião Norte de Minas Gerais, Brasil, 2016 (regressão de Poisson)

\begin{tabular}{lll}
\hline Variáveis & p-valor & RP (IC 95\%)* \\
\hline Computadores com internet nas UBS & 0,228 & \\
\hline Menos da metade & & $1,17(0,91-1,51)$ \\
\hline Quase todas ou todas & 1,00 \\
\hline Sabe como funciona o serviço de teleconsultoria & 0,595 & \\
\hline Não & & $1,16(0,67-2,00)$ \\
\hline Sim & 1,00 \\
\hline Recomenda o uso aos médicos da APS & 0,175 & \\
\hline Não & & $2,00(0,74-5,56)$ \\
\hline Sim & 1,00 \\
\hline Estratégias de motivação para uso do serviço & \\
\hline Não & 0,007 & $2,19(1,24-3,86)$ \\
\hline Sim & & 1,00 \\
\hline
\end{tabular}

Fonte: Os autores (2016).

(*) RP: Razão de Prevalência; IC95\%: Intervalo de Confiança de 95\%.

\section{Discussão}

Este estudo revelou que a macrorregião de saúde Norte de Minas Gerais apresenta características de uma região que se beneficiaria muito com a incorporação do uso do serviço de teleconsultoria na prática dos profissionais médicos que atuam na atenção primária, considerando as particularidades da região. Todavia, o cenário retratado revelou importantes barreiras estruturais à implementação desse serviço, como a falta 
de computadores com acesso à internet em todas as UBS e a qualidade insatisfatória de conexão da internet em alguns municípios, além de desconhecimento do serviço por parte de alguns gestores de saúde.

A teleconsultoria possibilitaria a comunicação rápida e direta dos médicos e trabalhadores de saúde da região com polos universitários de excelência, propiciando aos pacientes de municípios pequenos e remotos acesso a uma APS mais qualificada e resolutiva, rompendo o isolamento geográfico e social dessas localidades. Além disso, a teleconsultoria tem o potencial de reduzir encaminhamentos desnecessários ${ }^{12,13}$.

Os resultados de um estudo que analisou a utilização, efetividade e resolutividade das teleconsultorias realizadas pela Rede de Teleassistência de Minas Gerais (RTMG) evidenciaram que a teleconsultoria evitou o encaminhamento de pacientes em $80 \%$ dos $\operatorname{casos}^{12}$. Estudo realizado por Alkmim e outros ${ }^{14}$ também mostrou o potencial da teleconsultoria para reduzir encaminhamentos e ajudar a resolver problemas rotineiros de profissionais de saúde na atenção primária. Assim, em uma perspectiva de melhoria da assistência em saúde, a teleconsultoria configura-se como uma excelente oportunidade, principalmente para as regiões carentes e remotas, onde não se pode esperar uma cobertura especializada presencial ${ }^{15}$.

A precariedade da estrutura informacional nas UBS não é uma particularidade da macrorregião Norte de Minas Gerais. Estudo realizado a partir da análise do banco de dados resultante da fase de avaliação externa do primeiro ciclo do Programa de Melhoria do Acesso e da Qualidade da Atenção Básica (PMAQ$\mathrm{AB}$ ) apontou a necessidade de maiores investimentos na estrutura de informática nas UBS no Brasil, visto que das 13.843 unidades visitadas durante a avaliação externa, 98,3\% declararam ter, pelo menos, um computador em uso. Porém, apenas $45,1 \%$ possuíam acesso à internet ${ }^{16}$. Resultados de estudo realizado para identificar o grau de acesso e uso de tecnologias informacionais das equipes da Estratégia Saúde da Família (ESF) avaliadas pelo PMAQ-AB demonstraram a baixa eficiência da comunicação, com reduzida expansão da internet e de ferramentas informacionais: aproximadamente $22 \%$ de todas as equipes da ESF do Brasil utilizam a internet como ferramenta de comunicação e $8 \%$ a telessaúde como recurso no fluxo de comunicação. Essas informações reafirmam o grande desafio que é atingir os objetivos do acesso e uso de tecnologias informacionais na atenção básica do SUS ${ }^{17}$.

Nos países em desenvolvimento e em regiões remotas e carentes, onde a telessaúde tem aplicação direta na melhoria do acesso a serviços especializados, a qualidade da conexão com a internet às vezes é um fator limitante para a implementação de serviços virtuais ${ }^{18}$. Outro fator relevante é a falta de disponibilidade de computadores para realização de teleconsultorias nas UBS ${ }^{19}$.

De acordo com o presente estudo, o serviço de teleconsultoria não é utilizado pelos profissionais médicos que atuam na APS em quase um terço dos municípios da macrorregião de saúde Norte de Minas Gerais. Na análise dos fatores associados à não utilização do serviço de teleconsultoria, nenhuma variável relacionada às características do município mostrou-se associada. Em princípio, seria esperado que municípios menores, com menor IDHM e mais distantes dos municípios de referência para atenção especializada apresentassem maiores taxas de utilização do serviço, pois um estudo realizado por Marcolino e outros ${ }^{16}$, que analisou as teleconsultorias realizadas pela Rede de Telessaúde de Minas Gerais, no período de abril de 2007 a dezembro de 2012, identificou que a maior parte das teleconsultorias se originou de municípios com essas características.

É relevante destacar também que, mesmo nas análises bivariadas, nenhuma característica particular dos gestores municipais de saúde se mostrou associada, mas apenas aquelas relacionadas à interação do gestor com o serviço de teleconsultoria e, após a análise múltipla, apenas a não utilização de estratégias motivacionais por parte do gestor mostrou-se associada. Tal resultado vai ao encontro de um estudo realizado por Pessoa e outros ${ }^{19}$, realizado com profissionais que atuam na APS em Minas Gerais, que apontou que o apoio e a motivação do gestor local de saúde para o uso do serviço de teleconsultoria estão independentemente associados a uma maior utilização. Esses resultados ressaltam a necessidade de se promover maior aproximação entre gestores de saúde e serviços ofertados pela telessaúde, despertandolhes o interesse pelos serviços e sensibilizando-lhes quanto aos benefícios que os mesmos agregam ao 
sistema de saúde e à população, visto a importância dos gestores na integração dos profissionais de saúde ao serviço de teleconsultoria.

De acordo com o estudo realizado por Pessoa e outros ${ }^{19}$, o apoio e a motivação dos gestores locais de saúde representam o seu interesse na telessaúde e criam condições favoráveis para o uso da teleconsultoria. Assim, considerando que o não convencimento dos gestores de saúde em relação aos benefícios da telessaúde pode ter influência negativa na utilização do serviço de teleconsultoria ${ }^{19}$, capacitá-los em telessaúde, demonstrando seus benefícios clínicos e financeiros é essencial para alcançar o apoio efetivo dos mesmos e, consequentemente, a criação de condições para o uso de tecnologias de informação e comunicação na rotina dos serviços de saúde ${ }^{20}$. Isso é particularmente verdadeiro para países em desenvolvimento, onde os recursos para investimento são escassos e os gestores são envolvidos em outras prioridades da saúde, sofrendo pressões financeiras e administrativas, o que dificulta a difusão da telessaúde ${ }^{21}$.

Demonstrar o impacto positivo na relação custo-eficácia dos cuidados de saúde e da teleconsultoria pode convencer os gestores de saúde dos seus benefícios, levando-os a motivar outros profissionais de saúde $^{19}$. Para Jennett e outros ${ }^{22}$, é preciso convencer os gestores dos benefícios da telessaúde para se obter sucesso na sua utilização, sendo importante comprovar a relação de custo-efetividade. Cabe ressaltar que esse processo de convencimento não é fácil, principalmente porque a implementação do serviço de teleconsultoria nos municípios causa alterações nos modelos tradicionais de regulação assistencial, com o rompimento de referências em vigor e a eliminação de contratos para atendimentos em outros centros anteriormente estabelecidos, ferindo às vezes interesses regionais ${ }^{23}$. Além disso, para alguns gestores de pequenos municípios, um importante indicador de eficiência do sistema de saúde é o número de veículos, ambulâncias e motoristas para o transporte dos pacientes e, de certa forma, existe um ganho político associado ao procedimento ${ }^{24}$.

Os resultados do presente estudo devem ser interpretados considerando algumas limitações. Primeiramente, trata-se de um estudo transversal, que descreve apenas associação entre variáveis, sem possibilidade de estabelecer causalidade. Deve-se considerar ainda que as variáveis foram aferidas por meio de informação dos gestores municipais de saúde e não pelos profissionais médicos - usuários efetivos do serviço de teleconsultoria. É possível que a avaliação direta junto aos médicos da APS produza novos resultados. Contudo, apesar de tais limitações, trata-se do primeiro estudo realizado sobre a temática no Norte de Minas Gerais, região emblemática para áreas que podem se beneficiar com a utilização do serviço de teleconsultoria pelos profissionais médicos que atuam na atenção primária. Os achados permitem concluir que a incorporação do uso do serviço de teleconsultoria à prática dos médicos que atuam na APS nessa macrorregião é um grande desafio e demandam maior envolvimento dos gestores de saúde, além do atendimento às necessidades estruturais.

\section{Referências}

1. Giovanella L, Mendonça MHM, Almeida PF, Escorel S, Senna MCM, Fausto MCR, et al. Family health: limits and possibilities for an integral primary healthcare approach in Brazil. Ci Saúde Coletiva. 2009;14(3):783-94.

2. Bashshur RL, Reardon TG, Shannon GW. Telemedicine: a new health care delivery system. Annu Rev Public Health. 2000;21:613-37.

3. Hailey D, Ohinmaa A, Roine R. Study quality and evidence of benefit in recent assessments of telemedicine. J Telemed Telecare. 2004;10(6):318-24.

4. Pagliari C, Sloan D, Gregor P, Sullivan F, Detmer D, Kahan JP, et al. What is eHealth (4): a scoping exercise to map the field. J Med Internet Res. 2005;7(1):e9. 
5. Ministério da Saúde (BR); Universidade Federal do Rio Grande do Sul. Manual de Telessaúde para Atenção Básica/Atenção Primária à Saúde. Brasília; 2012.

6. Nerlich M, Balas EA, Schall T, Stieglitz SP, Filzmaier R, Asbach P, et al. Teleconsultation practice guidelines: report from G8 Global Health Applications Subproject 4. Telemed J E Health. 2002;8(4)411-8.

7. Alkmim MBM, Ribeiro ALP, Carvalho GGR, Abreu MP, Carvalho MLB. Success factors and difficulties for implementation of a telehealth system for remote villages: Minas telecardio project case in Brazil. J eHealth Technol Appl. 2007;5(2):197-202.

8. Instituto Brasileiro de Geografia e Estatística (BR). Censo demográfico 2010. Rio de Janeiro; 2012.

9. Ministério da Saúde (BR). Portaria no 2.546, de 27 de outubro de 2011. Redefine e amplia o Programa Telessaúde Brasil, que passa a ser denominado Programa Nacional Telessaúde Brasil Redes (Telessaúde Brasil Redes). DOU. out. 28;Seção 1.

10. Lee J, Tan CS, Chia KS. A practical guide for multivariate analysis of dichotomous outcomes. Ann Acad Med Singapore. 2009;38(8):714-9.

11. Programa das Nações Unidas para o Desenvolvimento; Instituto de Pesquisa Econômica Aplicada; Fundação João Pinheiro. Atlas do Desenvolvimento Humano no Brasil: índice de desenvolvimento humano municipal. Brasília; 2015.

12. Andrade MV, Maia AC, Cardoso CS, Alkmim MB, Ribeiro ALP. Custo-benefício do serviço de telecardiologia no Estado de Minas Gerais: projeto Minas Telecardio. Arq Bras Cardiol. 2011;97(4): 307-16.

13. Marcolino MS, Alkmim MB, Assis TGP, Sousa LAP, Ribeiro ALP. Teleconsultorias no apoio à atenção primária à saúde em municípios remotos no estado de Minas Gerais, Brasil. Rev Panam Salud Publica. 2014;35(5/6):345-52.

14. Alkmim MB, Marcolino MS, Figueira RM, Sousa L, Nunes MS, Cardoso CS, Ribeiro AL. Factors associated with the use of a teleconsultation system in Brazilian primary care. Telemed J E Health 2015;21(6):473-83.

15. Piropo TGN, Amaral HOS. Telessaúde, contextos e implicações no cenário baiano. Saúde debate 2015;39(104):279-87.

16. Neves TCCL, Montenegro LAA, Bittencourt SDA. Produção e registro de informações em saúde no Brasil: panorama descritivo através do PMAQ-AB. Saúde debate 2014;38(103):756-70.

17. Lima AMLD, Costa AA, Santos AF, Abreu DMX, Sobrinho DF, Lobato L, Jorge A, Machado ATGM. Acesso e uso de ferramentas informacionais na atenção básica à saúde no Brasil. In: $11^{\circ}$ Congresso Brasileiro de Saúde Coletiva; 2015 jul. 28-ago. 01; Universidade Federal de Goiás. Goiânia: Universidade Federal de Goiás; ABRASCO; 2015.

18. Alkmim MB, Figueira RM, Marcolino MS, Cardoso CS, Abreu MP, Cunha LR, Cunha DF, Antunes AP, Resende AGA, Resende ES, Ribeiro ALP. Improving patient access to specialized health care: the Telehealth Network of Minas Gerais, Brazil. Bull World Health Organ 2012;90: 373-8.

19. Pessoa CG, Sousa L, Ribeiro AL, Oliveira TB, Silva JLP, Alkmim MBM, Marcolino MS. Description of Factors Related to the use of the Teleconsultation System of a Large Telehealth Service in Brazil - the Telehealth Network of Minas Gerais. J Int Soc Telemed eHealth 2016; 4(e4):1-9.

20. Alkmim MBM, Maia JX, Marcolino MS, Cunha LR, Silva GAC, Figueira RM, et al. Nove anos de experiência em teleconsultorias para atenção primária. In: Mathias I, Monteiro A, organizadores. Gold Book Inovação Tecnológica em Educação e Saúde [Internet]. 2012 [citado em 2018 dez. 07]; 227-40. Disponível em: http://www.telessaude.uerj.br/goldbook/artigos/

21. Ohinmaa A, Hailey D, Roine R. Elements for assessment of telemedicine applications. Int J Technol Assess Health Care. 2001;17(2):190-202.

22. Jennett $P$, Jackson A, Ho K, Healy $T$, Kazanjian A, Woollard R, et al. The essence of telehealth readiness in rural communities: an organizational perspective. Telemed J E Health. 2005;11(2):137-45.

23. Nicolini, D. The work to make telemedicine work: a social and articulate view. Soc Sci Med. 2006;62(11):2754-67.

24. Alkmim MBM. Fatores associados à utilização de sistema de teleconsultoria na atenção primária de municípios remotos de Minas Gerais [dissertação]. Belo Horizonte: Universidade Federal de Minas Gerais; 2010. 